\title{
On Jacobi and Jacobi-Like Algorithms for a Parallel Computer
}

\author{
By Ahmed H. Sameh
}

\begin{abstract}
Many existing algorithms for obtaining the eigenvalues and eigenvectors of matrices would make poor use of such a powerful parallel computer as the ILLIAC IV. In this paper, Jacobi's algorithm for real symmetric or complex Hermitian matrices, and a Jacobi-like algorithm for real nonsymmetric matrices developed by P. J. Eberlein, are modified so as to achieve maximum efficiency for the parallel computations.
\end{abstract}

1. Introduction. With the advent of parallel computers, the study of computationally massive problems became economically possible. Such problems include, for example, solution of sets of partial differential equations over sizable grids, and multiplication, inversion, or determination of eigenvalues and eigenvectors of large matrices.

An example of a parallel computer is the ILLIAC IV.* This computer is essentially an array of coupled arithmetic units driven by instructions from a common control unit. Each of the arithmetic units, called processing elements (PE's), have 2048 words of 64-bit memory with an access time under 420 nanoseconds. Each PE is capable of 64-bit floating-point multiplication in about 550 nanoseconds. Two 32-bit floating-point operations may be performed in each PE in approximately the same times. The PE instruction set is similar to that of conventional machines with two exceptions. First, the PE's are capable of communicating data to four neighboring PE's by means of routing instructions. Second, the PE's are able to set their own mode registers to effectively disable or enable themselves. For a more detailed description of this system, the reader is referred to [2], [8], [9], [12].

The purpose of this paper is to introduce modified Jacobi and Jacobi-like algorithms for the computation of the eigenvalues and eigenvectors of large real symmetric or complex Hermitian matrices, and real nonsymmetric matrices, respectively, that are suitable for a parallel computer.

2. Jacobi's Algorithm. In the classical method of Jacobi (1846), [13], a real symmetric matrix is reduced to the diagonal form by a sequence of plane rotations $A_{k+1}=R_{k} A_{k} R_{k}^{t}(k=1,2, \cdots)$, where $A_{1}=A$ is the original matrix and each rotation $R_{k} \equiv R\left(p, q, \alpha_{p q}^{(k)}\right)$ in the $p, q$ plane through an angle $\alpha_{p q}^{(k)}$ eliminates the offdiagonal element $a_{p q}^{(k)}$ (and hence $a_{a p}^{(k)}$ ), and affects only elements in rows and columns $p$ and $q$. See the Appendix for the appropriate value of $\alpha_{p a}^{(k)}$ to annihilate the element $a_{p a}^{(k)}$. Because of symmetry, only the off-diagonal elements above the main diagonal are considered in what follows.

Received June 24, 1970, revised January 13, 1971.

AMS 1969 subject classifications. Primary 6540; Secondary 1525.

Key words and phrases. Parallel computers, ILLIAC IV, Jacobi's algorithm, Jacobi-like algorithm, orthogonal transformations, eigenvalues, eigenvectors, normal matrix.

* The ILLIAC IV system will soon be in operation. 
It is possible, however, to modify the present Jacobi algorithm for a parallel computer so as to eliminate more than one off-diagonal element. For example, for a matrix $A$ of order 4, if the orthogonal transformation $R$ is chosen as,

$$
R=\left[\begin{array}{rrrr}
c_{1} & 0 & s_{1} & 0 \\
0 & c_{2} & 0 & s_{2} \\
-s_{1} & 0 & c_{1} & 0 \\
0 & -s_{2} & 0 & c_{2}
\end{array}\right]
$$

where $c_{i}=\cos \alpha_{i}, s_{i}=\sin \alpha_{i}(i=1,2)$, then $R A R^{t}$ would have zero elements in positions $(1,3)$ and $(2,4)$ provided that the angles $\alpha_{1}$ and $\alpha_{2}$ are properly chosen. $\alpha_{1}$ and $\alpha_{2}$ are determined by $\left(a_{11}, a_{33}, a_{13}\right)$ and $\left(a_{22}, a_{44}, a_{24}\right)$, respectively.

Define $m$ by $[(n+1) / 2]$, where $n$ is the order of the matrix $A$ and $[x]$ is the greatest integer less than or equal to $x$. Let each $(2 m-1)$ orthogonal transformations be denoted by a sweep. Observing that there are $n(n-1) / 2$ off-diagonal elements, and that the maximum number of these elements which can be annihilated by an orthogonal transformation of the type (2.1) is [ $n / 2]$, then the modified Jacobi algorithm will attain maximum efficiency of parallel computation if the following two conditions are satisfied:

(i) Each orthogonal transformation $R_{k}$ should be constructed so as to annihilate [n/2] off-diagonal elements.

(ii) Each sweep should annihilate each off-diagonal element only once, i.e., each of the $(2 m-1)$ orthogonal transformations in a sweep should annihilate different $[n / 2]$ off-diagonal elements.

Several annihilation regimes that satisfy the above requirements are possible. Two different regimes are discussed below.

First Annihilation Regime. For a given sweep, each of the $(2 m-1)$ orthogona 1 matrices $R_{k}$ consists of the elements,

$$
\begin{aligned}
R_{p p}^{(k)}=R_{a q}^{(k)}=\cos \alpha_{p a}^{(k)} ; \quad R_{p q}^{(k)}=-R_{a p}^{(k)} & =\sin \alpha_{p q}^{(k)}, & & p<q, \\
& =-\sin \alpha_{p q}^{(k)}, \quad & & p>q,
\end{aligned}
$$

where $p$ and $q$ are sequences defined by

(a) for $k=1,2, \cdots, m-1$,

$$
\begin{array}{rlrl}
q & =m-k+1, m-k+2, \cdots, n-k, \\
p & =(2 m-2 k+1)-q, & & m-k+1 \leqq q \leqq 2 m-2 k, \\
& =(4 m-2 k)-q, & & 2 m-2 k<q \leqq 2 m-k-1, \\
& =n, & & 2 m-k-1<q,
\end{array}
$$

(b) for $k=m, m+1, \cdots, 2 m-1$,

$$
\begin{array}{rlrl}
q & =4 m-n-k, 4 m-n-k+1, \cdots, 3 m-k-1, \\
p & =n, & q<2 m-k+1, \\
& =(4 m-2 k)-q, & 2 m-k+1 \leqq q \leqq 4 m-2 k-1, \\
& =(6 m-2 k-1)-q, & 4 m-2 k-1<q .
\end{array}
$$


The remaining elements of $R_{k}$ are zero except for $n$ odd, then $R_{2 m-k, 2 m-k}^{(k)}=1$. For a given $k$, the angles $\alpha_{p q}^{(k)}$ are determined for all $(p, q)$ such that $\alpha_{p q}^{(k)}$ eliminates the element $a_{p q}^{(k)}$; see the Appendix.

Let $n=8$ and $k=2$, then the pairs $(p, q)$ are given by $\{(2,3) ;(1,4) ;(7,5) ;(8,6)\}$ and $R_{2}$ is of the form
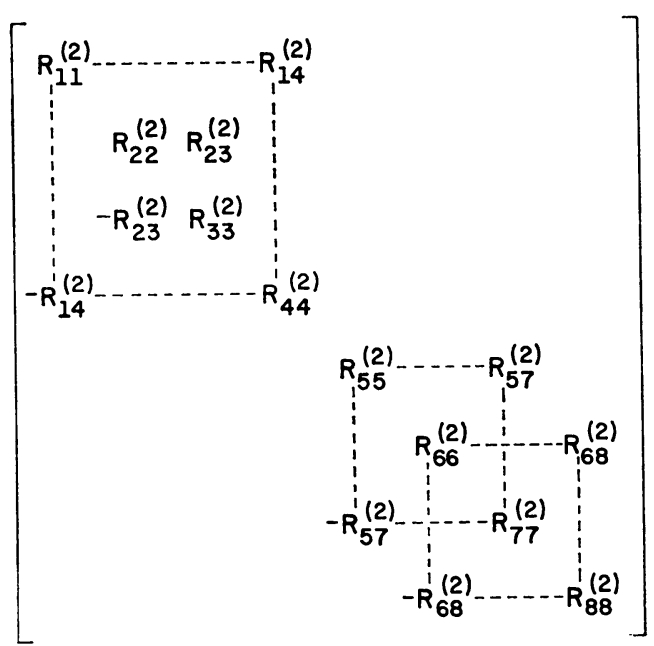

while for $k=7$ the pairs $(p, q)$ are $\{(8,1) ;(7,2) ;(6,3) ;(5,4)\}$ and $R_{7}$ is of the form

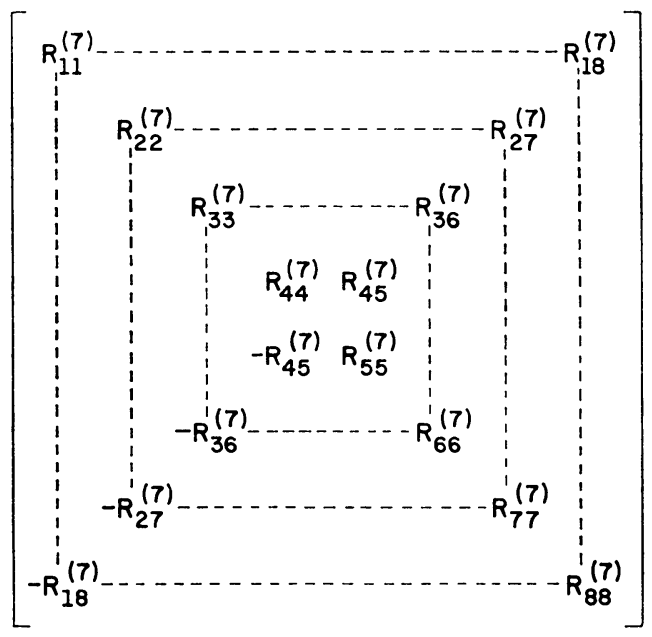

If the order of the matrix is odd, say $n=7$, then for $k=3$ the pairs $(p, q)$ are given by $\{(1,2) ;(7,3) ;(6,4)\}$ and $R_{3}$ is of the form 


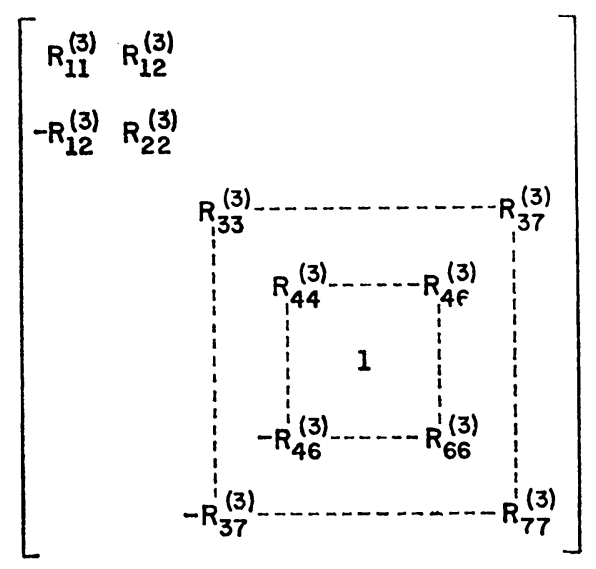

For example, in a given sweep, denoting each element eliminated in the $k$ th transformation by the integer $k$, the patterns of the annihilated elements for matrices of orders 16 and 15 are shown below.

$$
\begin{aligned}
& \text { * } 7 \text { (14) } 6 \text { (13) } 5 \text { (12) } 4 \text { (11) } 3 \text { (10) } 2 \text { (9) } 1 \text { (8) (15) } \\
& \text { * } 6 \text { (13) } 5 \text { (12) } 4 \text { (11) } 3 \text { (10) } 2 \text { (9) } 1 \text { (8) (15) (14) } \\
& \text { * } 5 \text { (12) } 4 \text { (11) } 3 \text { (10) } 2 \text { (9) } 1 \text { (8) (15) } 7 \text { (12) } \\
& \text { * } 4 \text { (12) } 3 \text { (10) } 2 \text { (9) } 1 \text { (8) (15) } 7 \text { (14):(12) } \\
& \text { * } 3 \text { (10) } 2 \text { (9) } 1 \text { (8) (15) } 7 \text { (14) } 6 \text { (111) } \\
& \text { * } 2 \text { (9) } 1 \text { (8) (15) } 7 \text { (14) } 6 \text { (13) (10) } \\
& \text { * } 1 \text { (8) (15) } 7 \text { (14) } 6 \text { (13) } 5 \text { (9) } \\
& \text { * (15) } 7 \text { (14) } 6 \text { (13) } 5 \text { (12) (8) } \\
& \text { * (14) } 6 \text { (13) } 5 \text { (12) } 4 \\
& \text { * (13) } 5 \text { (12) } 4 \text { (11) } 6 \\
& \text { * (12) } 4 \text { (12) } 35 \\
& \text { * (11) } 3 \text { (10) } 4 \\
& \text { * (10) } 2: 3 \\
& \text { * (9) } 2 \\
& \text { (15×15 }
\end{aligned}
$$

Second Annihilation Regime. This regime satisfies conditions (i) and (ii) for matrices of order $n=2^{\gamma}$, where $\gamma$ is an integer. The elements of each orthogonal transformation, in a given sweep, $R_{k}(k=1,2, \cdots, n-1)$ are given by (2.2). For $k=1$, $2, \cdots, n / 2$, the pairs $(p, q)$ are defined by

$$
\begin{aligned}
q & =2,4,6, \cdots, n, & & \\
p & =q+(n-2 k+1), & & q<2 k, \\
& =q-2 k+1, & & q \geqq 2 k .
\end{aligned}
$$

Let $n=8$ and $k=3$, then the pairs $(p, q)$ are $\{(5,2) ;(7,4) ;(1,6) ;(3,8)\}$ and $R_{3}$ 
is of the form

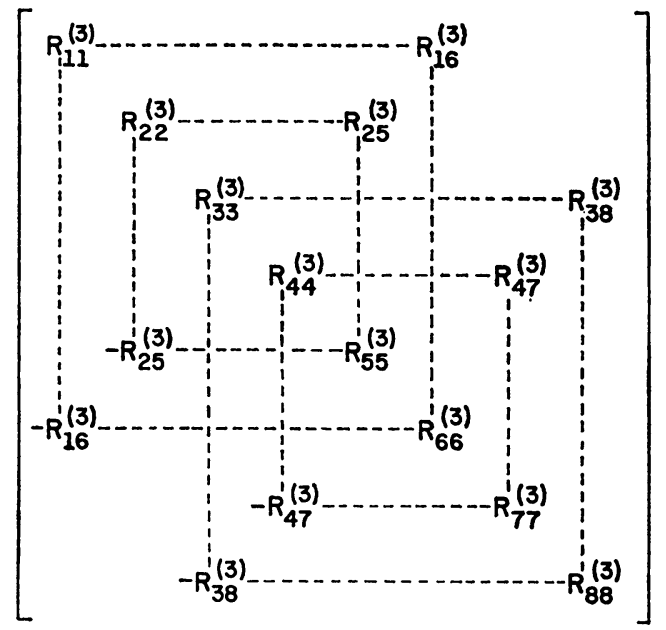

In order to construct the orthogonal transformations $R_{k}$ for $k=n / 2+1, n / 2+$ $2, \cdots, n-1$, consider the sequence $L=1,2, \cdots, \gamma-1$. For each value of $L$, there are $N=2^{\gamma-L-1}$ orthogonal matrices $R_{k}$ given by

$$
R_{k}=\operatorname{diag}\left(H_{1}^{(k)}, H_{2}^{(k)}, \cdots, H_{t}^{(k)}\right),
$$

where $t=2^{L-1}, k=n\left(1-2^{-L}\right)+l$, and $l=1,2, \cdots, N$. The sequences $p$ and $q$ for each $H_{M}^{(k)}(M=1,2, \cdots, t)$, are defined by

$$
\begin{aligned}
& p=i+4 N(M-1), \quad i=1,2, \cdots, 2 N, \\
& q=p+2(N+l-1)-2 N[O(1)],
\end{aligned}
$$

where

$$
\begin{aligned}
O(1) & =0, & & i+2(N+l-1) \leqq 4 N, \\
& =1, & & \text { otherwise. }
\end{aligned}
$$

Let $n=8, L=2$, and $l=1$, then $k=7$, and the pairs $(p, q)$ are given by $\{(1,3)$; $(2,4) ;(5,7) ;(6,8)\}$ and $R_{7}$ is of the form

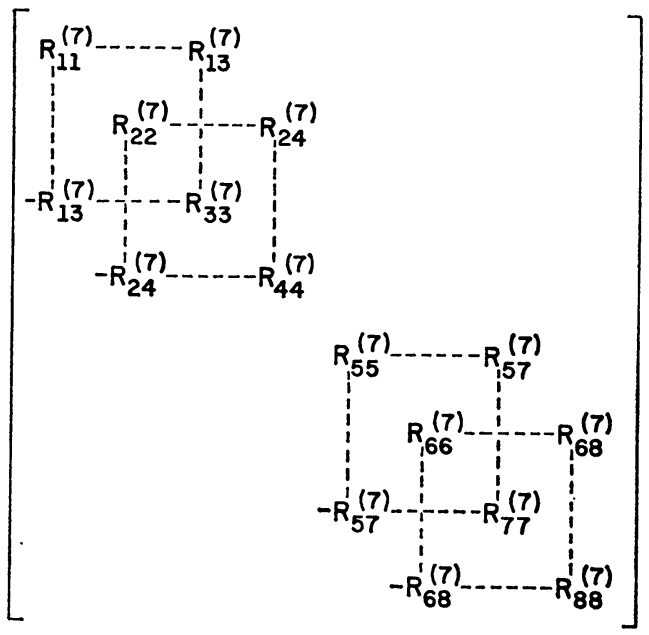


The pattern of the annihilated elements in one sweep for a matrix of order 16 is shown below, where those elements annihilated in the $k$ th transformation are denoted by the integer $k$.

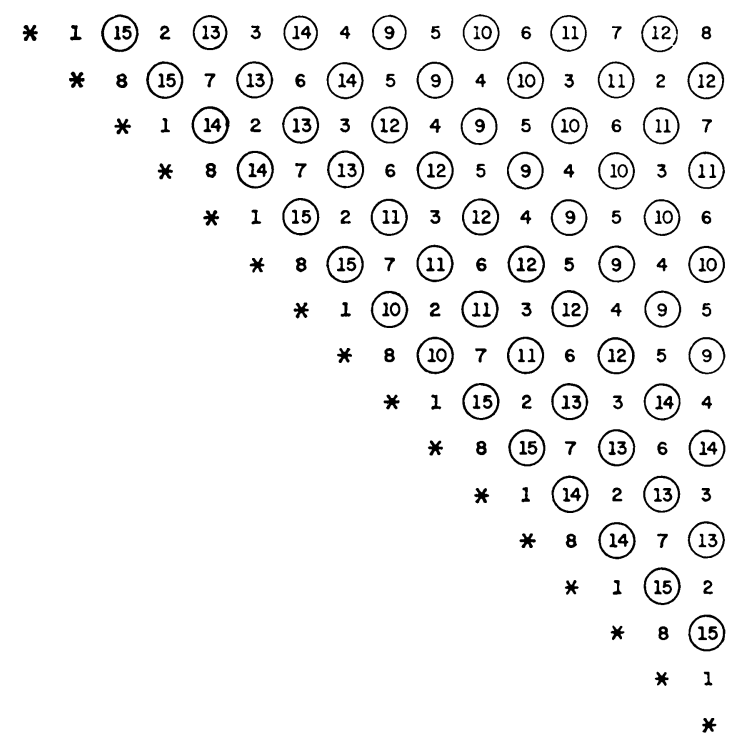

Using one quadrant of the ILLIAC IV (64 PE's), then for a $128 \times 128$ matrix, the 64 angles of each transformation are determined simultaneously, one angle per PE. Once the transformation matrix $R_{k}$ is determined, the matrix $A_{k+1}=R_{k} A_{k} R_{k}^{t}$ is computed in parallel [7]. Assuming that the matrix has converged, (using some criterion [13]), to the diagonal form after $u$ sweeps, or after $r-1=u(2 m-1)$ orthogonal transformations, then the diagonal elements of $A_{r}=W A W^{t}$ are taken to be the eigenvalues of $A$. The columns of $W^{t}=\left(V_{u} V_{u-1} \cdots V_{1}\right)^{t}$ are the corresponding eigenvectors, where for the $j$ th sweep $V_{j}=\prod_{k=1}^{2 m-1}\left(R_{k}\right)_{j}(j=1,2, \cdots, u)$.

A similar algorithm as that described above [11] has been programmed in ILLIAC IV assembly language and successfully tested on an ILLIAC IV execution simulator [1].

3. A Jacobi-Like Algorithm for Nonsymmetric Matrices. Eberlein [3], [4] showed that for an $n \times n$ matrix $A$, complex in general, there exists a matrix $U=$ $\prod_{l} U_{l}(k, m)$ generated from a sequence of two-dimensional transformations $U_{l}(k, m)$, where $(k, m)$ is the pivot pair, such that $A_{L}=U^{-1} A U$ is arbitrarily close to being normal, i.e., the matrix $\left(A_{L} A_{L}^{*}-A_{L}^{*} A_{L}\right)$ is arbitrarily small. At each stage of the iteration, based on the elements of the $k$ th and $m$ th rows and columns, the parameters of $U_{l}$ were chosen such that the decrement of the Euclidean norm of $A_{l}$ is given by

$$
N^{2}\left(A_{l}\right)-N^{2}\left(U_{l}^{-1} A_{l} U_{l}\right) \geqq[1 / 3 n(n-1)] \cdot N^{2}\left(A_{l} A_{l}^{*}-A_{l}^{*} A_{l}\right)
$$

where $N^{2}(A)=\sum_{i, i}\left|a_{i j}\right|^{2}$.

In this paper, the above algorithm has been modified for parallel computation. The transformations $U_{l}$ are $n$-dimensional, and their parameters are based on all the elements of the matrix $A_{l}$. A lower bound on the decrement of the Euclidean norm of $A_{l}$ is given by

$$
N^{2}\left(A_{l}\right)-N^{2}\left(U_{l}^{-1} A_{l} U_{l}\right) \geqq(1 / 4 n) N^{2}\left(A_{l} A_{l}^{*}-A_{l}^{*} A_{l}\right) .
$$


Once the matrix is practically normal, one can use the optimal procedure of Goldstine and Horwitz [5] for reducing it to the diagonal form; thus the eigenvalues and eigenvectors of $\boldsymbol{A}$ are obtained.

Since a nondiagonable matrix cannot be similar to a normal matrix, then this procedure yields its best results for diagonable matrices (see Example 7 in [3, p. 84]).

Let the original matrix $A$ be real, diagonable, and of an even order $n=2 r$ (if $n$ is odd $A$ is replaced by $\operatorname{diag}(A, \nu)$ of order $n+1)$, then it can be partitioned as follows

$$
A=\left[\begin{array}{cccc}
A_{11} & A_{12} & \cdots & A_{1 r} \\
A_{21} & A_{22} & \cdots & A_{2 r} \\
\vdots & \vdots & & \vdots \\
A_{r 1} & A_{r 2} & \cdots & A_{r r}
\end{array}\right]
$$

where each submatrix $A_{k m},(k, m=1,2, \cdots, r)$, is given by

$$
A_{k m}=\left[\begin{array}{cc}
a_{2 k-1,2 m-1} & a_{2 k-1,2 m} \\
a_{2 k, 2 m-1} & a_{2 k, 2 m}
\end{array}\right] .
$$

Let

$$
\begin{aligned}
& D_{k m}=\left(a_{2 k-1,2 m-1}-a_{2 k, 2 m}\right), \\
& E_{k m}=\left(a_{2 k-1,2 m}-a_{2 k, 2 m-1}\right), \\
& B_{k m}=\left(a_{2 k-1,2 m}+a_{2 k, 2 m-1}\right),
\end{aligned}
$$

and

$$
\begin{aligned}
& \kappa_{1}(A)=\sum_{k, m}\left(D_{k m}^{2}+E_{k m}^{2}\right), \\
& \kappa_{2}(A)=\sum_{k, m} D_{k m} E_{k m} .
\end{aligned}
$$

Assume also that $A$ has been scaled such that $N^{2}(A) \leqq 1$, and denote the matrix $\left(A A^{t}-A^{t} A\right)$ by $C$.

Lemma 1. Let $A^{\prime}=Q^{-1} A Q$, where $Q=\operatorname{diag}\left(S_{1}, S_{2}, \cdots, S_{r}\right)$, and $S_{1}=S_{2}=\cdots=S_{r}=S$ is given by

$$
S=\left[\begin{array}{cc}
\cosh \psi & \sinh \psi \\
\sinh \psi & \cosh \psi
\end{array}\right] .
$$

Define $\psi$ by

$$
\tanh 4 \psi=-2 \kappa_{2}(A) / \kappa_{1}(A) .
$$

Provided that $\kappa_{1}(A)>2\left|\kappa_{2}(A)\right|$, the following relation holds

$$
\Delta N^{2}(A) \geqq \kappa_{2}^{2}(A) / \kappa_{1}(A),
$$

where $\Delta N^{2}(A)=N^{2}(A)-N^{2}\left(A^{\prime}\right)$ is the decrement of the Euclidean norm of $A$.

Proof. The elements of each submatrix $A_{k m}^{\prime}=S^{-1} A_{k m} S$ are given by 


$$
\begin{aligned}
a_{2 k-1,2 m-1}^{\prime} & =a_{2 k-1,2 m-1} \cosh ^{2} \psi-a_{2 k, 2 m} \sinh ^{2} \psi+\frac{1}{2} E_{k m} \sinh 2 \psi, \\
a_{2 k, 2 m}^{\prime} & =-a_{2 k-1,2 m-1} \sinh ^{2} \psi+a_{2 k, 2 m} \cosh ^{2} \psi-\frac{1}{2} E_{k m} \sinh 2 \psi, \\
a_{2 k-1,2 m}^{\prime} & =\frac{1}{2} D_{k m} \sinh 2 \psi+a_{2 k-1,2 m} \cosh ^{2} \psi-a_{2 k, 2 m-1} \sinh ^{2} \psi, \\
a_{2 k, 2 m-1}^{\prime} & =-\frac{1}{2} D_{k m} \sinh 2 \psi-a_{2 k-1,2 m} \sinh ^{2} \psi+a_{2 k, 2 m-1} \cosh ^{2} \psi .
\end{aligned}
$$

Therefore,

$$
N^{2}\left(A_{k m}^{\prime}\right)=N^{2}\left(A_{k m}\right)+\left(D_{k m}^{2}+E_{k m}^{2}\right) \sinh ^{2} 2 \psi+D_{k m} E_{k m} \sinh 4 \psi
$$

and consequently,

$$
\Delta N^{2}\left(A_{k m}\right)=-D_{k m} E_{k m} \sinh 4 \psi-\frac{1}{2}\left(D_{k m}^{2}+E_{k m}^{2}\right)(\cosh 4 \psi-1) .
$$

Since $N^{2}(A)=\sum_{k, m} N^{2}\left(A_{k m}\right)$, then

$$
\Delta N^{2}(A)=-\frac{1}{2}(\cosh 4 \psi-1) \kappa_{1}(A)-(\sinh 4 \psi) \kappa_{2}(A) .
$$

A necessary condition for $\Delta N^{2}(A)$ to be an extremum with respect to $\psi$ is $(\partial / \partial \psi) \Delta N^{2}(A)=0$; this yields relation (3.6),

$$
\tanh 4 \psi=-2 \kappa_{2}(A) / \kappa_{1}(A) .
$$

From the definition (3.4), it is clear that $\kappa_{1}(A) \geqq 2\left|\kappa_{2}(A)\right|$. Excluding for the time being the case $\kappa_{1}(A)=2\left|\kappa_{2}(A)\right|$, then the second derivative of $\Delta N^{2}(A)$ with respect to $\psi$ evaluated for $\psi$ in (3.6) is given by

$$
-8 \kappa_{1}(A)\left[1-\left(4 \kappa_{2}^{2}(A) / \kappa_{1}^{2}(A)\right)\right](\cosh 4 \psi)
$$

and is less than zero. Thus, for the choice (3.6) of $\psi, \Delta N^{2}(A)$ achieves its maximum value,

$$
\Delta N^{2}(A)=\frac{1}{2} \kappa_{1}(A)\left[1-\left\{1-\left(4 \kappa_{2}^{2}(A) / \kappa_{1}^{2}(A)\right)\right\}^{1 / 2}\right]
$$

which vanishes only if $\kappa_{2}(A)=0$. Since one is considering the case $\kappa_{1}(A)>2\left|\kappa_{2}(A)\right|$, then by the binomial theorem,

$$
\left(1-4 \kappa_{2}^{2}(A) / \kappa_{1}^{2}(A)\right)^{1 / 2}=1-\frac{1}{2}\left(4 \kappa_{2}^{2}(A) / \kappa_{1}^{2}(A)\right)-\frac{1}{8}\left(4 \kappa_{2}^{2}(A) / \kappa_{1}^{2}(A)\right)^{2}-\cdots
$$

and (3.12) yields the relation (3.7). If $\kappa_{1}(A)=2\left|\kappa_{2}(A)\right|$, then from (3.10), $\Delta N^{2}(A)$ is given by $\frac{1}{2} \kappa_{1}(A)\left[1-\left\{(1 \pm \tanh 4 \psi) /\left(1-\tanh ^{2} 4 \psi\right)^{1 / 2}\right\}\right]$. Choosing $\tanh 4 \psi=$ $\mp\left(1-\epsilon^{2}\right) /\left(1+\epsilon^{2}\right)$, where $\epsilon$ is a small number, then $\Delta N^{2}(A)=\frac{1}{2}(1-\epsilon) \kappa_{1}(A)$ which is greater than zero.

Lemma 2. Let $A^{\prime}=P^{t} A P$, where $P$ is the orthogonal transformation,

$$
P=\operatorname{diag}\left(T_{1}, T_{2}, \cdots, T_{r}\right)
$$

in which

$$
T_{k}=\left[\begin{array}{rr}
\cos \varphi_{k} & \sin \varphi_{k} \\
-\sin \varphi_{k} & \cos \varphi_{k}
\end{array}\right] \quad(k=1,2, \cdots, r)
$$

Then, if $\varphi_{k}$ is determined by

$$
\tan 2 \varphi_{k}=\frac{c_{2 k-1,2 k-1}-c_{2 k, 2 k}}{2 c_{2 k-1,2 k}},
$$


where $c_{i j}$ are the elements of the matrix $C=A A^{t}-A^{t} A$,

$$
\kappa_{2}^{2}\left(A^{\prime}\right) \geqq \frac{1}{2 n} N^{2}(C) .
$$

Proof. The $2 \times 2$ diagonal submatrices $C_{k k}$ of the matrix $C$ can be expressed as

$$
C_{k k}=\sum_{m=1}^{r}\left[A_{k m} A_{k m}^{t}-A_{m k}^{t} A_{m k}\right], \quad k=1,2, \cdots, r .
$$

Therefore,

$$
\sum_{k=1}^{r} C_{k k}=\sum_{k, m=1}^{r}\left[A_{k m} A_{k m}^{t}-A_{k m}^{t} A_{k m}\right]
$$

where

$$
\left(A_{k m} A_{k m}^{t}-A_{k m}^{t} A_{k m}\right)=\left[\begin{array}{cc}
E_{k m} B_{k m} & -D_{k m} E_{k m} \\
-D_{k m} E_{k m} & -E_{k m} B_{k m}
\end{array}\right] .
$$

Equating the off-diagonal elements of the left- and right-hand sides of (3.19),

$$
\sum_{k=1}^{r} c_{2 k-1,2 k}=-\sum_{k, m} D_{k m} E_{k m}=-\kappa_{2}(A)
$$

Consequently, if the orthogonal matrix $P$ is chosen such that the off-diagonal elements $c_{2 k-1,2 k}$, for all $k$, attain their maximum positive values, then the inequality (3.17) is achieved. To show that, consider the matrix $C^{\prime}=A^{\prime} A^{\prime t}-A^{\prime t} A^{\prime}$. Since $A^{\prime}=P^{t} A P$, then $C^{\prime}=P^{t} C P$, and the elements of the diagonal submatrices $C_{k k}^{\prime}=$ $T_{k}^{t} C_{k} T_{k}$ are given by

$$
\begin{aligned}
c_{2 k-1,2 k}^{\prime} & =c_{2 k-1,2 k} \cos 2 \varphi_{k}+\frac{1}{2}\left(c_{2 k-1,2 k-1}-c_{2 k, 2 k}\right) \sin 2 \varphi_{k}, \\
c_{2 k-1,2 k-1}^{\prime} & =c_{2 k-1,2 k-1} \cos ^{2} \varphi_{k}+c_{2 k, 2 k} \sin ^{2} \varphi_{k}-c_{2 k-1,2 k} \sin 2 \varphi_{k}, \\
c_{2 k, 2 k}^{\prime} & =c_{2 k-1,2 k-1} \sin ^{2} \varphi_{k}+c_{2 k, 2 k} \cos ^{2} \varphi_{k}+c_{2 k-1,2 k} \sin 2 \varphi_{k}, \quad \text { and } \\
c_{2 k, 2 k-1}^{\prime} & =c_{2 k-1,2 k}^{\prime} .
\end{aligned}
$$

Hence, for $c_{2 k-1,2 k}^{\prime}$ to be an extremum, (3.16) must hold. Also, for the choice (3.16) of $\varphi_{k}$, the second derivative of $c_{2 k-1,2 k}^{\prime}$ with respect to $\varphi_{k}$ is given by

$$
-\left(h^{2} / c_{2 k-1,2 k}\right) \cos 2 \varphi_{k},
$$

where $h=\left[4 c_{2 k-1,2 k}^{2}+\left(c_{2 k-1,2 k-1}-c_{2 k, 2 k}\right)^{2}\right]^{1 / 2}$. As a result if $\cos 2 \varphi_{k}$ is of the same sign as $c_{2 k-1,2 k}, c_{2 k-1,2 k}^{\prime}$ attains its maximum value. Restricting $\varphi_{k}$ to the interval $[0, \pi]$, the elements of $T_{k}$ are given by

$$
\sin ^{2} \varphi_{k}=\frac{1}{2}-\left(c_{2 k-1,2 k} / h\right), \quad \cos ^{2} \varphi_{k}=\frac{1}{2}+\left(c_{2 k-1,2 k} / h\right),
$$

in which $\sin \varphi_{k}>0$ and $\cos \varphi_{k}$ is of the same sign as $\left(c_{2 k-1,2 k-1}-c_{2 k, 2 k}\right)$. The maximum value of $c_{2 k-1,2 k}^{\prime}$ turns out to be $\frac{1}{2} h$, and

$$
c_{2 k-1,2 k-1}^{\prime}=c_{2 k, 2 k}^{\prime}=\frac{1}{2}\left(c_{2 k-1,2 k-1}+c_{2 k, 2 k}\right) \text {. }
$$

Excluding the case when $c_{2 k-1,2 k-1}=c_{2 k, 2 k}$ and $c_{2 k-1,2 k}=0$, which results in $T_{k}$ 
being the identity matrix and hence $c_{2 k-1,2 k}^{\prime}=0$, then from (3.21) one obtains the inequality

$$
\kappa_{2}^{2}\left(A^{\prime}\right)>\sum_{k=1}^{r} c_{2 k-1,2 k}^{\prime 2}
$$

Assuming that $\sum_{k=1}^{r} c_{2 k-1,2 k}^{\prime 2} \geqq(1 / 2 n) N^{2}\left(C^{\prime}\right)$, then, from the fact that the Euclidean norm is invariant under orthogonal transformations and from (3.25), one obtains relation (3.17).

From Lemmas 1 and 2, it can be seen that in order to obtain the largest possible value of $\Delta N^{2}(A)$, the matrix $A$ should be subjected to the orthogonal transformation $M^{t} A M$ where $M$ is a permutation matrix determined as follows: Let $A^{\prime \prime}=M^{t} A M$ and $C^{\prime \prime}=A^{\prime \prime} A^{\prime \prime t}-A^{\prime \prime t} A^{\prime \prime}$, then $M$ is chosen such that each $2 \times 2$ diagonal submatrix $C_{k k}^{\prime \prime}$ has an element $c_{2 k-1,2 k}^{\prime \prime}$ of at least average absolute value of all the offdiagonal elements of $C^{\prime \prime}$ if any, and/or the difference $\left(c_{2 k-1,2 k-1}^{\prime \prime}-c_{2 k, 2 k}^{\prime \prime}\right)$ different from zero. For example, in order to bring the off-diagonal element $c_{u v},(u<v)$, of maximum absolute value in the position $(1,2), M$ is given by $I_{1 u}, I_{2}$, where $I_{i i}=I-$ $\left(e_{i}-e_{i}\right)\left(e_{i}-e_{i}\right)^{t}$. Essentially, $I_{i j}^{i} A I_{i j}$ has the $i$ th and $j$ th rows and columns of $A$ exchanged.

After the matrix $A$ is "prepared" by the transformation $M, A^{\prime}=P^{t} A^{\prime \prime} P$ will produce a matrix $C^{\prime}$ whose off-diagonal elements $c_{2 k-1,2 k}^{\prime}$ are of such magnitudes that $\sum_{k=1}^{r} c_{2 k-1,2 k}^{\prime 2}$ is at least equal to $(1 / 2 n) N^{2}(C)$.

THEOREM. Let $A=A_{1}$ be a diagonable matrix with an even order $n=2 r$ and $N^{2}(A) \leqq 1$. Let $A_{l+1}=U_{l}^{-1} A_{l} U_{l}$, where $U_{l}=M_{l} P_{l} Q_{l}$. If these transformations are defined as follows:

(i) $M_{l}$ is chosen as discussed above.

(ii) $P_{l}=\operatorname{diag}\left(T_{1}^{(l)}, T_{2}^{(l)}, \cdots, T_{r}^{(l)}\right)$ in which

$$
T_{k}^{(l)}=\left[\begin{array}{cc}
\cos \varphi_{k}^{(l)} & \sin \varphi_{k}^{(l)} \\
-\sin \varphi_{k}^{(l)} & \cos \varphi_{k}^{(l)}
\end{array}\right]
$$

with

$$
\tan 2 \varphi_{k}^{(l)}=\frac{c_{2 k-1,2 k-1}^{(l)}-c_{2 k, 2 k}^{(l)}}{2 c_{2 k-1,2 k}^{(l)}}
$$

(iii) $Q_{l}=\operatorname{diag}\left(S_{1}^{(l)}, S_{2}^{(l)}, \cdots, S_{r}^{(l)}\right)$ in which

$$
S_{1}^{(l)}=S_{2}^{(l)}=\cdots=S_{r}^{(l)}=\left[\begin{array}{cc}
\cosh \psi_{l} & \sinh \psi_{l} \\
\sinh \psi_{l} & \cosh \psi_{l}
\end{array}\right]
$$

with

$$
\tanh 4 \psi_{l}=-2 \kappa_{2}\left(A_{l}^{\prime}\right) / \kappa_{1}\left(A_{l}^{\prime}\right)
$$

where

$$
A_{l}^{\prime}=\left(M_{l} P_{l}\right)^{t} A_{l}\left(M_{l} P_{l}\right)
$$

Then, $\lim _{l \rightarrow \infty} N^{2}\left(C_{l}\right)=0$.

Proof. With no loss of generality, assume that $M_{l}=I$. By Lemma $2, \kappa_{2}^{2}\left(A_{l}^{\prime}\right) \geqq$ $(1 / 2 n) N^{2}\left(C_{l}\right)$. From (3.3), $\left(D_{k m}^{(l)}\right)^{2}+\left(E_{k m}^{(l)}\right)^{2} \leqq 2 N^{2}\left(A_{k m}^{(l)}\right)$, then (3.4) yields, $\kappa_{1}\left(A_{l}\right) \leqq$ 
$2 N^{2}\left(A_{l}\right) \leqq 2$. Since the Euclidean norm is invariant under orthogonal transformations, then $\kappa_{1}\left(A_{l}^{\prime}\right) \leqq 2$, and hence by Lemma 1 ,

$$
\Delta N^{2}\left(A_{l}\right) \geqq \kappa_{2}^{2}\left(A_{l}^{\prime}\right) / \kappa_{1}\left(A_{l}^{\prime}\right) \geqq \frac{1}{4 n} N^{2}\left(C_{l}\right) .
$$

But since $N^{2}\left(A_{l}\right)$ is a decreasing monotone function bounded below by $\sum_{i}\left|\lambda_{i}\right|^{2}$, where $\lambda_{i}$ are the eigenvalues of $A$, [10], then $\Delta N^{2}\left(A_{l}\right) \rightarrow 0$ as $l \rightarrow \infty$. Hence $N^{2}\left(C_{l}\right) \rightarrow 0$, and $A_{l}$ is arbitrarily close to being normal.

Let $A$ be a $128 \times 128$ matrix. Using one quadrant of the ILLIAC IV (64 PE's), the matrix can be stored in memory such that for a given $m$ the $2 \times 2$ submatrices $A_{k m}(k=1,2, \cdots, 64)$ are assigned to the $m$ th PE. Once the matrix $C$ is determined by parallel multiplication and stored in the same way, i.e., the $k$ th PE contains the submatrix $C_{k k}$, the 64 angles $\varphi_{k}$ can then be determined simultaneously. Also for each $k$ the submatrices $A_{k m}^{\prime}=T_{k}^{t} A_{k m} T_{m}$ are computed simultaneously for all $m$, hence the updated matrix $A^{\prime}=P^{t} A P$ is computed with all the PE's working. Similarly the quantities $D_{k m}^{\prime}, E_{k m}^{\prime}$, and $B_{k m}^{\prime}$ of the submatrices $A_{k m}^{\prime}$, and consequently the submatrices $S^{-1} A_{k m}^{\prime} S$ are computed with full efficiency. This part of the algorithm has been coded and successfully tested on the ILLIAC IV simulator [1].

Once the matrix $A$ is reduced to a matrix $\widetilde{A}$ which is practically normal, then for any diagonal submatrix

$$
\left[\begin{array}{ll}
\tilde{a}_{p p} & \tilde{a}_{p q} \\
\tilde{a}_{a p} & \tilde{a}_{a q}
\end{array}\right]
$$

either $\tilde{a}_{p q}=\tilde{a}_{a p}$; or $\tilde{a}_{p q}=-\tilde{a}_{q p}$ and $\tilde{a}_{p p}=\tilde{a}_{q q}$, to within a reasonable computational error. The matrix $\tilde{A}$ is reduced to the diagonal form by the unitary transformations $V_{j}^{*} \tilde{A}_{j} V_{j}(j=1,2,3, \cdots)$, where $V_{j}=\prod_{k=1}^{2 m-1}\left(R_{k}\right)_{j}$, as in Section 2, is the transformation matrix of the $j$ th sweep. For each off-diagonal element $\tilde{a}_{p e}$ or $\tilde{a}_{a p}$ above the diagonal, the elements of the diagonal submatrices of $R_{k}$ are given by

(a) $\tilde{a}_{p q}^{(k)}=\tilde{a}_{a p}^{(k)}$;

the elements $R_{p p}^{(k)}, R_{a q}^{(k)}, R_{p q}^{(k)}$, and $R_{q p}^{(k)}$ are determined as in Section 2.

(b) $\tilde{a}_{p q}^{(k)}=-\tilde{a}_{q p}^{(k)}$ and $\tilde{a}_{p p}^{(k)}=\tilde{a}_{a q}^{(k)}$;

$$
R_{p p}^{(k)}=R_{a q}^{(k)}=\frac{1}{\sqrt{ } 2} ; \quad R_{p a}^{(k)}=R_{a p}^{(k)}=\frac{i}{\sqrt{ } 2}, \text { where } i=(-1)^{1 / 2}
$$

Denoting the resulting matrix by $\Lambda=Y^{-1} A Y$, the diagonal elements of $\Lambda$ are then the eigenvalues of $A$, and the columns of the matrix $Y=\left(\prod_{l} U_{l}\right)\left(\prod_{i} V_{i}\right)$ are the corresponding eigenvectors.

4. Acknowledgment. The author would like to thank Professor Daniel L. Slotnick, Director of the Center for Advanced Computation, University of Illinois, for introducing him to the subject of parallel computation and for providing encouragement and guidance throughout the investigation. Special thanks go to the referee for his valuable comments and criticism of the presentation.

Appendix. The orthogonal matrix $R\left(p, q, \alpha_{p a}^{(k)}\right)$ differs from the identity matrix by a $2 \times 2$ diagonal submatrix whose elements are 
(A.1)

$$
R_{p p}=R_{e q}=\cos \alpha_{p a}^{(k)} ; \quad R_{p q}=-R_{a p}=\sin \alpha_{p q}^{(k)}
$$

where $p<q$. In order to eliminate the off-diagonal element $a_{p q}^{(k)}$, the angle $\alpha_{p a}$ is chosen such that

$$
\tan 2 \alpha_{p q}^{(k)}=\frac{2 a_{p q}^{(k)}}{a_{p p}^{(k)}-a_{a q}^{(k)}}
$$

in which $\alpha_{p a}^{(k)}$ is restricted by $\left|\alpha_{p a}^{(k)}\right| \leqq \pi / 4$, [6]. Let

$$
t_{k}=\left|2 a_{p q}^{(k)}\right|, \quad x_{k}=\left|a_{p p}^{(k)}-a_{a q}^{(k)}\right|, \quad y_{k}=\left(t_{k}^{2}+x_{k}^{2}\right)^{1 / 2} ;
$$

then

$$
\cos ^{2} \alpha_{p q}^{(k)}=\frac{1}{2}\left(1+\frac{x_{k}}{y_{k}}\right) ; \quad \sin ^{2} \alpha_{p q}^{(k)}=\frac{1}{2}\left(1-\frac{x_{k}}{y_{k}}\right) .
$$

Since $\left|\alpha_{p q}^{(k)}\right| \leqq \pi / 4$, then $\cos \alpha_{p q}^{(k)}$ will always be taken positive and $\sin \alpha_{p q}^{(k)}$ will be of the same sign as $\left[2 a_{p q}^{(k)} /\left(a_{p p}^{(k)}-a_{a q}^{(k)}\right)\right]$.

Center for Advanced Computation

University of Illinois

Urbana, Illinois 61801

1. W. Bernhard, ILLIAC IV Codes for Jacobi and Jacobi-Like Algorithms, A Forthcoming Center for Advanced Computation Document, University of Illinois, Urbana, Illinois.

2. R. L. Davis, "The ILLIAC IV processing element," IEEE Trans. Computers, v. C-18, 1969 , pp. 800-816.

3. P. J. EbERLEIN, "A Jacobi-like method for the automatic computation of eigenvalues and eigenvectors of an arbitrary matrix," J. Soc. Indust. Appl. Math., v. 10, 1962, pp. 74-88. MR 25 \#2699.

4. P. J. Eberlein \& J. Boothroyd, "Solution to the eigenproblem by a norm reducing Jacobi type method," Numer. Math., v. 11, 1968. pp. 1-12.

5. H. H. GoldstiNe \& L. P. HoRwitz, "A procedure for the diagonalization of normal matrices," J. Assoc. Comput. Mach., v. 6, 1959, pp. 176-195. MR 21 \#426.

6. P. Henrici, "On the speed of convergence of cyclic and quasicyclic Jacobi methods for computing eigenvalues of Hermitian matrices," J. Soc. Indust. Appl. Math., v. 6, 1958, pp. 144-162. MR 20 \#2084.

7. M. Knowles, B. Okawa, Y. Muroka \& R. Wilhelmson, Matrix Operations on ILLIAC IV, ILLIAC IV Document \#52, Dept. of Computer Science, University of Illinois, Urbana, Illinois, 1967.

8. D. J. KUCK,"ILLIAC IV software and application programming," IEEE Trans. Computers, v. C-17, 1968, pp. 758-770.

9. D. C. McINTYRE, "An introduction to the ILLIAC IV computer," Datamation, v. 16 , No. 4, 1970, pp. $60-67$.

10. L. MIRSKY, "On the minimization of matrix norms," Amer. Math. Monthly, v. 65, 1958, pp. 106-107. MR 20 \#3169.

11. A. SameH \& L. HaN, Eigenvalue Problems, ILlIAC IV Document \#127, Dept. of Computer Science, University of Illinois, Urbana, Illinois, 1968.

12. D. L. SLOtNICK ET AL., "The ILLIAC IV computer," IEEE Trans. Computers, v. C-17, 1968, pp. 746-757.

13. J. H. Wilkinson, The Algebraic Eigenvalue Problem, Clarendon Press, Oxford, 1965. MR 32 \#1894. 